\title{
Linear Wave Interaction with a Vertical Cylinder of Arbitrary Cross Section: An Asymptotic Approach
}

\author{
N. B. Dişibüyük ${ }^{1}$; A. A. Korobkin²; and O. Yilmaz ${ }^{3}$
}

\begin{abstract}
An asymptotic approach to the linear problem of regular water waves interacting with a vertical cylinder of an arbitrary cross section is presented. The incident regular wave was one-dimensional, water was of finite depth, and the rigid cylinder extended from the bottom to the water surface. The nondimensional maximum deviation of the cylinder cross section from a circular one plays the role of a small parameter of the problem. A fifth-order asymptotic solution of the problem was obtained. The problems at each order were solved by the Fourier method. It is shown that the first-order velocity potential is a linear function of the Fourier coefficients of the shape function of the cylinder, the second-order velocity potential is a quadratic function of these coefficients, and so on. The hydrodynamic forces acting on the cylinder and the water surface elevations on the cylinder are presented. The present asymptotic results show good agreement with numerical and experimental results of previous investigations. Long-wave approximation of the hydrodynamic forces was derived and used for validation of the asymptotic solutions. The obtained values of the forces are exact in the limit of zero wave numbers within the linear wave theory. An advantage of the present approach compared with the numerical solution of the problem by an integral equation method is that it provides the forces and the diffracted wave field in terms of the coefficients of the Fourier series of the deviation of the cylinder shape from the circular one. The resulting asymptotic formula can be used for optimization of the cylinder shape in terms of the wave loads and diffracted wave fields. DOI: 10.1061/ (ASCE)WW.1943-5460.0000407. @ 2017 American Society of Civil Engineers.
\end{abstract}

Author keywords: Linear water waves; Noncircular vertical cylinder; Asymptotic analysis; Wave loads.

\section{Introduction}

Prediction of wave forces is important to engineers for the design of offshore and coastal structures. Floating airports, bridge pylons, semi-submersibles, and tension leg platforms are typical examples of such structures. For large-scale structures, one should take the diffraction effects into account. The potential wave theory is usually used to estimate the wave loads.

The wave-body interaction is a three-dimensional and nonlinear problem with a position of the free surface of the liquid that is unknown in advance and an unknown wetted surface of the body. The problem can be linearized for waves of small amplitude compared with the water depth, wave length, and linear size of the body. Within the linear theory of water waves, the free surface boundary conditions are linearized and imposed on the equilibrium level of the water surface. Viscous effects and surface tension of the liquid are important for short water waves with relatively high frequency. For large dimensions of offshore structures and moderate wave length, both viscous and capillary effects can be neglected at leading order. The resulting linear problem of wave theory is additionally simplified if the water depth is constant and the structure is a

${ }^{1}$ Ph.D. Student and Research Assistant, Dept. of Mathematics, Dokuz Eylul Univ., Tnaztepe Campus, Buca, Izmir 35390, Turkey (corresponding author). ORCID: https://orcid.org/0000-0002-8339-1304. E-mail: bugurcan.ruzgar@deu.edu.tr

${ }^{2}$ Professor, School of Mathematics, Univ. of East Anglia, Norwich, U.K. NR4 7TJ. E-mail: a.korobkin@uea.ac.uk

${ }^{3}$ Professor, Dept. of Mathematics, Izmir Institute of Technology, Urla, Izmir 35430, Turkey. E-mail: oguzyilmaz@iyte.edu.tr

Note. This manuscript was submitted on July 8, 2016; approved on February 21, 2017; published online on June 16, 2017. Discussion period open until November 16, 2017; separate discussions must be submitted for individual papers. This paper is part of the Journal of Waterway, Port, Coastal, and Ocean Engineering, (C) ASCE, ISSN 0733-950X. vertical cylinder extending from the flat sea bottom to the free surface. Such cylinders represent legs of offshore platforms and piles of offshore wind turbines. Offshore platforms are used for exploration of oil and gas from under the seabed and processing. A general offshore structure has a deck that is supported by deck legs. The hydrodynamic forces acting on these legs are of major concern to engineers because the design of the legs is dominated by wave loads. In many applications, the cylinders have circular cross sections, but not necessarily.

One of the first studies of diffraction of plane water waves by stationary obstacles with vertical sides was performed by Havelock (1940) for water of infinite depth. Results were obtained for cylinders of circular and parabolic sections. Cylinders of ship forms were also studied by Havelock (1940) using some approximations with applications to a ship advancing in waves. The draught of the ship was assumed infinite. Havelock (1940) noticed that, for periodic linear water waves and obstacles with vertical sides, both time and the vertical coordinates can be separated from the problem, and the original problem of water waves can be reduced to the two-dimensional problem of plane sound waves diffracted by the two-dimensional rigid body representing the cross section of the vertical cylinder. Then, known results from diffraction problems of sound and electromagnetic waves can be transferred and applied to the problem of water waves diffracted by a vertical cylinder. MacCamy and Fuchs (1954) extended this approach to water of finite constant depth and a surface-piercing vertical circular cylinder.

Chen and Mei (1971) solved the water wave diffraction problem for a vertical elliptic cylinder. The elliptic cylindrical coordinates and the method of separating variables were used to find the velocity potential in terms of an infinite series of Mathieu functions. In another study, Chen and Mei (1973) investigated the same problem using long-wave approximation. Numerical results were also presented for a ship-like body. Williams (1985) used two different 
methods to solve the diffraction problem for elliptic vertical cylinders. One method used the two-term asymptotic expansions of the exact solution for the forces and moments acting on the elliptic cylinder with small eccentricity. The second method was the integral equation method. It was concluded that the asymptotic method gives good results for small wave numbers. In a recent study by Liu et al. (2016), wave diffraction by a uniform bottom-mounted cylinder with an arbitrary cross section was numerically studied. The velocity potential was sought in the form suggested by MacCamy and Fuchs (1954) for a circular cylinder. However, the coefficients in the Fourier series for diffracted waves were determined by using the body boundary condition for the noncircular cylinder. In this numerical method, the body boundary condition was satisfied approximately by the Galerkin method. Fourier series were used to represent the cross sections of the cylinders and the free surface elevation. As a practical application of this numerical method, the wave forces and wave run-up on quasi-elliptic caisson foundations of a cross-strait bridge pylon were investigated. These numerical results are used in the present paper for validation of the authors' asymptotic solutions.

There are two main approaches to the numerical treatment of the diffraction problem of cylinders with arbitrary cross section. One is the integral equation method developed by Hwang and Tuck (1970) in the investigation of harbor resonance. Isaacson (1978) applied this method to calculations of wave forces on cylinders used in offshore structures. The method is based on source or source-dipole distribution and Green's theorem. The resulting Fredholm integral equation (the second approach) for the velocity potential is solved by discretizing the cylinder contour into small segments. This method was also used by Mansour et al. (2002) and Wu and Price (1991). Wu and Price (1991) calculated wave drift forces acting on multiple vertical cylinders of arbitrary cross sections. The boundary element method was developed by $\mathrm{Au}$ and Brebbia (1983) for the diffraction problem of vertical cylinders. This method is based on the Galerkin weighted residual formulation. After obtaining the integral equation, the boundary of the cylinder is discretized into boundary elements that are chosen to be constant, linear, or quadratic. The boundary element method was applied to the cylinders of circular, elliptic, and square cross sections. Au and Brebbia (1983) obtained the wave forces acting on a square cylinder and compared their results with the experimental and numerical results of Mogridge and Jamieson (1976). The agreement between the numerical, experimental, and theoretical predictions of the hydrodynamic forces acting on the square cylinder was shown to be fairly good. Approximation of equivalent circular radius was used by Mogridge and Jamieson (1976). In this approximation, the horizontal hydrodynamic force acting on a vertical cylinder is approximated by the force acting on the circular cylinder with the same area as its cross section. The boundary element method of Au and Brebbia (1983) was used by Zhu and Moule (1994) in the problem of short-crested wave interaction with vertical cylinders with arbitrary cross sections. The boundary element method for the diffraction problem of vertical and horizontal cylinders is explained in detail by Wrobel et al. (1985).

Numerical methods such as the integral equation method and the boundary element method can be used to solve the diffraction problem for vertical cylinders with an arbitrary section. However, in some cases, these numerical methods are not preferable, such as when evaluating free surface integrals in the second-order diffraction problem of vertical cylinders (Eatock Taylor and Hung 1987). The free surface integral converges slowly, and the values of the first-order potential have to be evaluated many times, which is not possible with the integral equation methods. Also, the integral equation methods require quite fine discretization of the boundary of the cylinder, which could be tedious and is the source of errors. The method of the present paper, which was originally proposed by Mei et al. (2005), can deal with geometries with arbitrary cross sections with little effort.

In this paper, linear water waves scattered by a vertical cylinder with an arbitrary cross section extending from the sea bottom to the free surface in water of finite depth were studied by asymptotic methods. The nondimensional maximum deviation of the cylinder cross section from a circular one plays the role of a small parameter of the problem. A fifth-order asymptotic solution of the problem was obtained. Numerical calculations of the diffracted velocity potential, the forces acting on the cylinder, and the diffracted wave field were reduced to operations with the Fourier coefficients of the shape function, which describes the cross section of the cylinder, and the velocity potentials on the cylinder surface at each order of approximation. It is shown that the first-order velocity potential is a linear function of the Fourier coefficients of the shape function of the cylinder, the second-order velocity potential is a quadratic function of these coefficients, and so on. The obtained solution makes it possible to formulate and solve two practical problems in terms of the Fourier coefficients of the shape function: optimization of the shape of the cylinder and identification of the cylinder shape by using a measured wave field far from the cylinder. The asymptotic approach of this paper was applied to calculations of the hydrodynamic forces acting on elliptic, quasi-elliptic, and square cylinders. The comparisons of the asymptotic forces with available numerical and experimental results by others demonstrate good accuracy of the present approach. Long-wave approximation of the hydrodynamic forces is obtained and used for validation of the asymptotic solution.

Note that Mei et al. (2005) were concerned with the leading order corrections to the forces caused by small deviation of a vertical elliptic cylinder from the circular one. A similar perturbation approach was used by Mansour et al. (2002) for vertical cylinders with a cosine-type radial perturbation of the cylinder cross section. The leading order corrections to the forces were obtained and compared to the numerical results by the integral equation method. It was shown that the agreement is good for small perturbation amplitude. In contrast to the perturbation analysis by Mansour et al. (2002), the present asymptotic approach is not restricted to a particular cylinder shape, and a fifth-order approximation of the solution was obtained. This paper shows that the fifth-order asymptotic solution makes it possible to consider even such noncircular cylinders as square ones and to obtain accurate results in terms of the hydrodynamic forces.

The present asymptotic approach can be extended to truncated vertical cylinders and oscillating rigid and elastic cylinders of arbitrary cross sections, as well as to submerged horizontal cylinders in plane incident waves. The diffraction problem of a truncated vertical cylinder with a circular cross section of radius $a$ was solved by Garrett (1971). In that paper, both the incident and diffracted waves were expanded in Bessel functions in the interior region $(r<a)$ and in the exterior region $(r>a)$, and then these two solutions and the derivatives of the solutions were matched at the boundary $(r=a)$. Black et al. (1971) used a variational formulation and a theorem due to Haskind to calculate wave forces on a stationary body using only far-field properties. Yeung (1981) used the same method to solve the radiation problem for a truncated vertical cylinder with a circular cross section. In the case of deep water, the multipole expansions are usually convenient to describe the velocity potential for wave diffraction and radiation. Ursell (1950) used a series of complex 
potential functions arising from multipoles at the center of the cylinder to solve the problem of the generation of surface waves by a submerged circular cylinder. Thorne (1953) investigated the motion arising from line and point singularities using multipole expansion method in deep and shallow waters. Two-dimensional multipoles were developed in a systematic way for submerged and floating cylinders by Eatock Taylor and $\mathrm{Hu}$ (1991). The application to arbitrary body shapes was accomplished by coupling the multipole expansion with a boundary integral method.

The outline of the paper is as follows. Mathematical formulation of the problem and its solution for a vertical circular cylinder are given in the next section. The fifth-order asymptotic solution of the problem is described in the "Vertical Cylinders with Nearly Circular Cross Sections" section. Each approximation of this asymptotic solution is obtained by operating with the Fourier coefficients of the shape function and the velocity potentials of the lowerorder approximations. The asymptotic approach is applied to elliptic cylinders in waves, and the obtained results are compared with the numerical results of Williams (1985) in the "Hydrodynamic Force on Elliptic Vertical Cylinder" section. In the next section, the present approach is applied to square cylinders, and the obtained results are compared with experimental results by Mogridge and Jamieson (1976) in terms of the horizontal forces acting on square cylinders. In the section "Hydrodynamic Force on Quasi-Elliptic Vertical Cylinder," the results of the present asymptotic method are compared with the three-dimensional solution of the Navier-Stokes equations by Wang et al. (2011). The wave force and run-up values for cylinders with circular, elliptic, quasi-elliptic, and square cross sections with the same cross-sectional area are compared. In the next section, the wave force and wave run-up on cylinders with cosine-type perturbations of their cross sections are studied and compared with the numerical results by Mansour et al. (2002) and Liu et al. (2016). Asymptotic behavior of wave forces for long waves is studied in the section "Long Wave Approximation of Wave Forces" for cylinders with arbitrary cross sections. The findings of the analysis are summarized and conclusions are drawn in the last section of this paper.

\section{Formulation of the Problem}

Diffraction of two-dimensional water waves by a vertical cylinder with an almost circular cross section is studied within the linear wave theory. The problem is formulated in a polar coordinate system $(r, \theta, z)$ where the $z$-axis points vertically upward. The plane $z=-h$ corresponds to the sea bottom, and the plane $z=0$ corresponds to the mean level of water surface. The rigid cylinder extends from the sea bottom to the free surface. The cross section of the vertical cylinder is described by the equation $r=R[1+\varepsilon f(\theta)]$, where $R$ is the mean radius of the cylinder, and $\varepsilon$ is a small nondimensional parameter of the problem. The top view of the studied configuration is shown in Fig. 1. The smooth and bounded function $f(\theta)$ describes the deviation of the shape of the cylinder from the circular one. A one-dimensional incident wave of amplitude $A$ and wave frequency $\omega$ propagates at angle $\alpha$ to the positive $x$-axis from $-\infty$ toward the cylinder. Within the linear wave theory (Mei et al. 2005), the wave field is described by a velocity potential $\Phi(r, \theta, z, t)$. For a vertical cylinder with an arbitrary cross section, the velocity potential is expressed only through the propagating wave mode

$$
\Phi(r, \theta, z, t)=\operatorname{Re}\left\{\frac{g A}{\omega} \frac{\cosh [k(z+h)]}{\cosh (k h)} \phi(r, \theta) e^{-i \omega t}\right\}
$$

where $i=\sqrt{-1}$; and $\operatorname{Re}\{\mathcal{A}\}=$ real part of a complex number $(\mathcal{A})$. The complex-valued function $\phi(r, \theta)$ satisfies the Helmholtz equation

$$
\phi_{r r}+\frac{1}{r} \phi_{r}+\frac{1}{r^{2}} \phi_{\theta \theta}+k^{2} \phi=0 \quad\{r>R[1+\varepsilon f(\theta)]\}
$$

in the flow region, the far-field condition

$$
\phi \sim e^{i k r \cos (\theta-\alpha)} \quad(r \rightarrow \infty)
$$

and the boundary condition on the surface of the cylinder

$$
\frac{\partial \phi}{\partial n}=0 \quad\{r=R[1+\varepsilon f(\theta)]\}
$$

where $\mathbf{n}=$ unit outward normal vector to the surface of the cylinder; $g=$ gravitational acceleration; $k=$ wave number; $k=2 \pi / \lambda$; and $\lambda=$ length of the incident wave. The wave number $k$ is related to the wave frequency $\omega$ by the dispersion relation $\omega^{2}=g k \tanh (k h)$, $k>0$.

The hydrodynamic force $\mathbf{F}(t)=\left(\mathcal{F}_{x}, \mathcal{F}_{y}\right)$ acting on the vertical cylinder is obtained by integration of the dynamic pressure $p(r, \theta, z, t)=-\rho \partial \Phi / \partial t$ over the wetted part of the cylinder

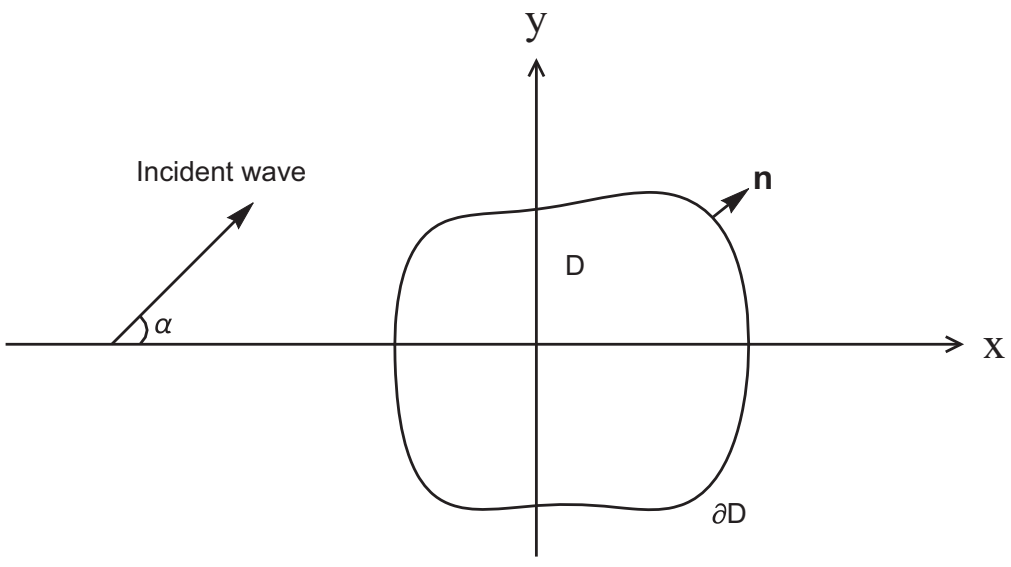

Fig. 1. Top view of the problem configuration 


$$
\begin{aligned}
\mathbf{F}(t) & =-\int_{-h}^{0} \int_{\partial D} p \mathbf{n} d s d z \\
& =-\rho g A \frac{\tanh (k h)}{k} \operatorname{Re}\left[i \int_{\partial D} \phi(r, \theta) \mathbf{n} d s e^{-i \omega t}\right]
\end{aligned}
$$

where $\partial D=$ boundary of the cylinder cross section; $r=R[1+\varepsilon f(\theta)]$; and $d s=$ small element of this boundary. The nondimensional force scaled with $\rho g A \pi a^{2} \tanh (k h)$ is denoted by tilde. Here, $a$ is a characteristic dimension of the vertical cylinder cross section, which can be different from $R$. The components of the nondimensional force are given by

$$
\begin{aligned}
\tilde{\mathcal{F}}_{x}(t)= & \operatorname{Re}\left(\tilde{F}_{x} e^{-i \omega t}\right), \quad \tilde{\mathcal{F}}_{y}(t)=\operatorname{Re}\left(\tilde{F}_{y} e^{-i \omega t}\right) \\
\tilde{F}_{x}= & \frac{-i R}{\pi k a^{2}} \int_{0}^{2 \pi} \phi(R[1+\varepsilon f(\theta)], \theta)\left\{\varepsilon f^{\prime}(\theta) \sin \theta\right. \\
& +[1+\varepsilon f(\theta)] \cos \theta\} d \theta \\
\tilde{F}_{y}= & \frac{-i R}{\pi k a^{2}} \int_{0}^{2 \pi} \phi(R[1+\varepsilon f(\theta)], \theta)\left\{-\varepsilon f^{\prime}(\theta) \cos \theta\right. \\
& +[1+\varepsilon f(\theta)] \sin \theta\} d \theta
\end{aligned}
$$

Note that the phases of $\tilde{F}_{x}$ and $\tilde{F}_{y}$ depend on the position of the origin $(O)$ of the coordinate system (Fig. 1), but the moduli $\left|\tilde{F}_{x}\right|$ and $\left|\tilde{F}_{y}\right|$ are independent of the coordinate system.

The elevation of the free surface $z=\eta(r, \theta, t)$ is related to the unknown potential $\phi(r, \theta)$ by the linear kinematic boundary condition $\eta_{t}=\Phi_{z}(r, \theta, 0, t)$, which gives

$$
\eta(r, \theta, t)=\operatorname{Re}\left[i A \phi(r, \theta) e^{-i \omega t}\right]
$$

The far-field condition Eq. (3) and Eq. (9) provide the assumed shape of the incident wave $\eta_{I}(r, \theta, t)$

$$
\eta_{I}(r, \theta, t)=A \sin [\omega t-k r \cos (\theta-\alpha)]
$$

where $\eta_{I}(0, \theta, t)=A \sin (\omega t)$ at the origin of the coordinate system.

The maximum elevation of the water surface at the cylinder per the wave period is known as the wave run-up $\triangle(\theta)$. The wave runup is scaled in this paper with the wave height $2 A$. Eq. (9) yields $\triangle(\theta) / 2 A=|\phi(r, \theta)| / 2$, where $r=R[1+\varepsilon f(\theta)]$. The wave runup and its dependence on the shape of the cylinder are important in the design of offshore structures, where the wave run-up should not exceed the elevation of the wet deck of an offshore structure above the mean water level. Wave run-up on offshore structures could be much higher than that predicted by the linear wave theory (De Vos et al. 2007; Lykke Andersen et al. 2011). Nonlinear waves with a steep front or breaking in front of the structure produce a thin runup sheet and spray near the structure, increasing the run-up. The run-up can be also affected by aeration of water near the structure due to the wave breaking, in particular. Many nonlinear physical effects near the structure are not included in the present linear model. However, it can be shown that these effects provide small contributions to the hydrodynamic structure (Iafrati and Korobkin 2006; Korobkin and Malenica 2007; Korobkin 2008) and the diffracted wave field.

Eqs. (2)-(4) for an arbitrary vertical cylinder can be solved numerically only. The analytical solution is well known for the circular cylinder $r=R$ (MacCamy and Fuchs 1954). In this study, this solution corresponds to the leading-order velocity potential of Eqs. (2)-(4) as $\varepsilon \rightarrow 0$

$$
\phi_{0}(r, \theta)=\sum_{m=0}^{\infty} \epsilon_{m} i^{m}\left[J_{m}(k r)-\frac{J_{m}^{\prime}(k R)}{H_{m}^{(1)^{\prime}}(k R)} H_{m}^{(1)}(k r)\right] \cos [m(\theta-\alpha)]
$$

where $\epsilon_{m}=$ Neumann symbol; $\epsilon_{0}=1, \epsilon_{m}=2$ for $m \geq 1, J_{m}(r)=$ Bessel functions of the first kind with order $m ; H_{m}^{(1)}(r)=$ Hankel functions of the first kind corresponding to outward-propagating cylindrical waves; and prime = derivatives with respect to the argument. By using the Wronskian identity $J_{m}(r) H_{m}^{(1)^{\prime}}(r)-J_{m}^{\prime}(r)$ $H_{m}^{(1)}(r)=2 i /(\pi r)$, the potential $\phi_{0}(r, \theta)$ on the surface of the cylinder is given by

$$
\phi_{0}(R, \theta)=\frac{2 i}{\pi k R} \sum_{m=0}^{\infty} \frac{\epsilon_{m} i^{m}}{H_{m}^{(1)^{\prime}}(k R)} \cos [m(\theta-\alpha)]
$$

Here

$$
\frac{2 i}{\pi k R H_{m}^{(1)^{\prime}}(k R)} \sim \sqrt{\frac{2}{\pi m}} e^{-m \log \left(\frac{2 m}{e k R}\right)}
$$

as $m \rightarrow \infty$. Therefore, Eq. (12) converges exponentially, and only a few terms are needed to calculate the potential $\phi_{0}(R, \theta)$ and its derivatives in $\theta$ with good accuracy.

Eqs. (12) and (7) provide the total nondimensional hydrodynamic force acting on the circular cylinder in the incident regular wave with $\alpha=0^{\circ}$ and $a=R$ (Mei et al. 2005)

$$
\tilde{F}_{x}=\frac{4 i}{\pi(k R)^{2} H_{1}^{(1)^{\prime}}(k R)}
$$

The force formula similar to Eq. (14) for $\varepsilon>0$ and a given function $[f(\theta)]$ describing the cross section of a noncircular vertical cylinder are determined here.

\section{Vertical Cylinders with Nearly Circular Cross Sections}

Asymptotic methods are used to find an approximate solution for Eqs. (2)-(4) as $\varepsilon \rightarrow 0$. The derivatives $\partial \phi / \partial \theta$ and $\partial \phi / \partial r$ in the boundary condition [Eq. (4)] on the surface of the cylinder

$$
\begin{aligned}
& \frac{\partial \phi}{\partial r}(R[1+\varepsilon f(\theta)], \theta)-\frac{\varepsilon f^{\prime}(\theta)}{R[1+\varepsilon f(\theta)]^{2}} \frac{\partial \phi}{\partial \theta}(R[1+\varepsilon f(\theta)], \theta) \\
& \quad=0
\end{aligned}
$$

are approximated by their Taylor series up to $\mathcal{O}\left(\varepsilon^{5}\right)$ at $r=R$, and then the fifth-order asymptotic expansion of the potential $\phi(r, \theta)$

$$
\begin{aligned}
\phi(r, \theta)= & \phi_{0}(r, \theta)+\varepsilon \phi_{1}(r, \theta)+\varepsilon^{2} \phi_{2}(r, \theta)+\varepsilon^{3} \phi_{3}(r, \theta) \\
& +\varepsilon^{4} \phi_{4}(r, \theta)+\mathcal{O}\left(\varepsilon^{5}\right)
\end{aligned}
$$

is substituted in the boundary condition. The resulting approximation of Eq. (4) is 


$$
\begin{aligned}
& \phi_{0, r}+\varepsilon\left[\phi_{1, r}+R f(\theta) \phi_{0, r r}-\frac{f^{\prime}(\theta)}{R} \phi_{0, \theta}\right] \\
& +\varepsilon^{2}\left[\phi_{2, r}+R f(\theta) \phi_{1, r r}-\frac{f^{\prime}(\theta)}{R} \phi_{1, \theta}+\frac{R^{2} f^{2}(\theta)}{2} \phi_{0, r r r}+\frac{2 f(\theta) f^{\prime}(\theta)}{R} \phi_{0, \theta}-f(\theta) f^{\prime}(\theta) \phi_{0, r \theta}\right] \\
& +\varepsilon^{3}\left[\begin{array}{c}
\phi_{3, r}+R f(\theta) \phi_{2, r r}-\frac{f^{\prime}(\theta)}{R} \phi_{2, \theta}+\frac{R^{2} f^{2}(\theta)}{2} \phi_{1, r r r}+\frac{2 f(\theta) f^{\prime}(\theta)}{R} \phi_{1, \theta}-f(\theta) f^{\prime}(\theta) \phi_{1, r \theta} \\
+2 f^{2}(\theta) f^{\prime}(\theta) \phi_{0, r \theta}+\frac{R^{3} f^{3}(\theta)}{6} \phi_{0, r r r r}-\frac{3 f^{2}(\theta) f^{\prime}(\theta)}{R} \phi_{0, \theta}-\frac{R f^{2}(\theta) f^{\prime}(\theta)}{2} \phi_{0, r r \theta}
\end{array}\right] \\
& +\varepsilon^{4}\left[\begin{array}{c}
\phi_{4, r}+R f(\theta) \phi_{3, r r}-\frac{f^{\prime}(\theta)}{R} \phi_{3, \theta}+\frac{R^{2} f^{2}(\theta)}{2} \phi_{2, r r r}-f(\theta) f^{\prime}(\theta) \phi_{2, r \theta}+\frac{2 f(\theta) f^{\prime}(\theta)}{R} \phi_{2, \theta} \\
-\frac{3 f^{2}(\theta) f^{\prime}(\theta)}{R} \phi_{1, \theta}-\frac{R f^{2}(\theta) f^{\prime}(\theta)}{2} \phi_{1, r r \theta}+2 f^{2}(\theta) f^{\prime}(\theta) \phi_{1, r \theta}+\frac{R^{3} f^{3}(\theta)}{6} \phi_{1, r r r r} \\
-3 f^{3}(\theta) f^{\prime}(\theta) \phi_{0, r \theta}+R f^{3}(\theta) f^{\prime}(\theta) \phi_{0, r r \theta}-\frac{R^{2} f^{3}(\theta) f^{\prime}(\theta)}{6} \phi_{0, r r r \theta}+\frac{R^{4} f^{4}(\theta)}{24} \phi_{0, r r r r} \\
+\frac{4 f^{3}(\theta) f^{\prime}(\theta)}{R} \phi_{0, \theta}
\end{array}\right]=\mathcal{O}\left(\varepsilon^{5}\right)
\end{aligned}
$$

where the functions $\phi_{n}(r, \theta)$ and their derivatives are calculated at $r=R$. At the leading order as $\varepsilon \rightarrow 0$, Eq. (16) provides $\phi_{0, r}(R, \theta)=0$. This is the boundary condition that leads to the solution Eq. (11) for the circular cylinder. At the first order, Eq. (16) gives

$$
\phi_{1, r}(R, \theta)=\frac{f^{\prime}(\theta)}{R} \phi_{0, \theta}(R, \theta)-R f(\theta) \phi_{0, r r}(R, \theta)
$$

The unknown potentials $\phi_{n}(r, \theta), n=0,1,2,3,4$, in Eq. (15) satisfy Eq. (2). This equation is used, in particular, to calculate the second derivative $\phi_{0, r r}$ and to write Eq. (17) in terms of $\phi_{0}(R, \theta)$ given by Eq. (12) and its derivatives in $\theta$

$$
\phi_{1, r}(R, \theta)=\frac{1}{R} f(\theta) \phi_{0, \theta \theta}+\frac{1}{R} f^{\prime}(\theta) \phi_{0, \theta}+R k^{2} f(\theta) \phi_{0}(R, \theta)
$$

Note that the wave number $k$ now appears in the boundary condition for the potential $\phi_{1}(r, \theta)$.

Equating the terms in Eq. (16) with $\varepsilon^{2}, \varepsilon^{3}$, and $\varepsilon^{4}$ to zero, the boundary conditions for the potentials $\phi_{2}, \phi_{3}$, and $\phi_{4}$ are obtained, respectively. These boundary conditions have the form $(n=1,2,3,4)$

$$
\phi_{n, r}(R, \theta)=G_{n}(\theta)
$$

where $G_{n}(\theta)=$ sums of the products of the functions $f(\theta), f^{\prime}(\theta), \phi_{0}(R, \theta), \ldots, \phi_{n-1}(R, \theta)$ and derivatives of the potentials $\phi_{0}(R, \theta), \ldots, \phi_{n-1}(R, \theta)$ in $\theta$. Starting from the solution Eq. (12) for the circular cylinder and a given function $f(\theta)$, the right-hand side $G_{1}(\theta)$ in Eq. (18) is calculated, and then the outward-propagating wave solution $\phi_{1}(r, \theta)$ of Eq.
(2) subject to the boundary condition Eq. (18) on the circular cylinder $r=R$ is determined. By using the obtained potential $\phi_{1}(r, \theta), G_{2}(\theta)$ is calculated and $\phi_{2}(r, \theta)$ is determined, and so on. The boundary value problems for the potentials $\phi_{n}(r, \theta)$ are identical and differ only by functions $G_{n}(\theta)$ in the body boundary condition [Eq. (19)]. By using the Fourier series of the functions $G_{n}(\theta)$

$$
G_{n}(\theta)=\frac{1}{2} G_{n 0}^{(c)}+\sum_{m=1}^{\infty} G_{n m}^{(c)} \cos (m \theta)+G_{n m}^{(s)} \sin (m \theta)
$$

the potentials are given by

$$
\begin{aligned}
\phi_{n}(r, \theta)= & \frac{1}{2} G_{n 0}^{(c)} \frac{H_{0}^{(1)}(k r)}{k H_{0}^{(1)^{\prime}}(k R)}+\sum_{m=1}^{\infty}\left[G_{n m}^{(c)} \cos (m \theta)\right. \\
& \left.+G_{n m}^{(s)} \sin (m \theta)\right] \frac{H_{m}^{(1)}(k r)}{k H_{m}^{(1)^{\prime}}(k R)}
\end{aligned}
$$

In particular, $\phi_{n}(R, \theta)$ and their derivatives are obtained in the form of their Fourier series. Calculations of the functions $G_{n}(\theta)$ and their Fourier coefficients are reduced to multiplication and summation of Fourier series. If the coefficients in the Fourier series of the function $f(\theta)$ are known

$$
f(\theta) \sim \frac{f_{0}^{(c)}}{2}+\sum_{m=1}^{\infty} f_{m}^{(c)} \cos (m \theta)+f_{m}^{(s)} \sin (m \theta)
$$

and using the Fourier series [Eq. (12)] of $\phi_{0}(R, \theta)$, the derivatives $f^{\prime}(\theta), \phi_{0, \theta}(R, \theta)$, and $\phi_{0, \theta \theta}(R, \theta)$ are calculated by differentiating Eqs. (12) and (22) term by term, and then the Fourier coefficients of the right-hand side in Eq. (18) can be determined. Finally, the solution $\phi_{1}(r, \theta)$ is given by Eq. (21). Similar 
arguments are applied to the higher-order problems for $\phi_{2}, \phi_{3}$, and $\phi_{4}$. It is seen that the asymptotic solution [Eq. (15)] of the problem is obtained by operating with the Fourier coefficients of the potentials $\phi_{n}(R, \theta)$ and the function $f(\theta)$, which describe the shape of the vertical cylinder. Summation and differentiation of Fourier series are straightforward operations. The multiplication of two Fourier series

$$
\begin{aligned}
& g(\theta) \sim \frac{a_{0}}{2}+\sum_{m=1}^{\infty} a_{m} \cos (m \theta)+b_{m} \sin (m \theta) \\
& h(\theta) \sim \frac{\alpha_{0}}{2}+\sum_{m=1}^{\infty} \alpha_{m} \cos (m \theta)+\beta_{m} \sin (m \theta)
\end{aligned}
$$

provides the Fourier series

$$
g(\theta) h(\theta) \sim \frac{A_{0}}{2}+\sum_{m=1}^{\infty} A_{m} \cos (m \theta)+B_{m} \sin (m \theta)
$$

where (Fichtenholtz 2001)

$$
\begin{aligned}
& A_{n}=\frac{a_{0} \alpha_{n}}{2}+\frac{1}{2} \sum_{m=1}^{\infty}\left[a_{m}\left(\alpha_{m+n}+\alpha_{m-n}\right)+b_{m}\left(\beta_{m+n}+\beta_{m-n}\right)\right] \\
& B_{n}=\frac{a_{0} \beta_{n}}{2}+\frac{1}{2} \sum_{m=1}^{\infty}\left[a_{m}\left(\beta_{m+n}-\beta_{m-n}\right)-b_{m}\left(\alpha_{m+n}-\alpha_{m-n}\right)\right]
\end{aligned}
$$

$$
\beta_{m-n}=-\beta_{n-m} \text { and } \alpha_{m-n}=\alpha_{n-m} \text { if } m-n<0
$$

Calculations of the components $\tilde{F}_{x}$ and $\tilde{F}_{y}$ of the hydrodynamic force acting on the vertical cylinder and the diffracted waves far from the cylinder can also be reduced to operations with the Fourier coefficients of the function $f(\theta)$ and the potentials $\phi_{n}(R, \theta)$. For example, the integrand of $\tilde{F}_{x}$ in Eq. (7) can be approximated as

$$
\begin{aligned}
\phi & (R[1+\varepsilon f(\theta)], \theta)\left\{\varepsilon f^{\prime}(\theta) \sin \theta+[1+\varepsilon f(\theta)] \cos \theta\right\} \\
& =\sum_{n=0}^{4} \varepsilon^{n} S_{n}(\theta)+\mathcal{O}\left(\varepsilon^{5}\right)
\end{aligned}
$$

where

$$
S_{n}(\theta)=\frac{1}{2} S_{n 0}^{(c)}+\sum_{m=1}^{\infty}\left[S_{n m}^{(c)} \cos (m \theta)+S_{n m}^{(s)} \sin (m \theta)\right]
$$

The nondimensional $x$-component of the force is given by

$$
\tilde{F}_{x}=-\frac{i R}{k a^{2}}\left[S_{00}^{(c)}+\varepsilon S_{10}^{(c)}+\varepsilon^{2} S_{20}^{(c)}+\varepsilon^{3} S_{30}^{(c)}+\varepsilon^{4} S_{40}^{(c)}\right]+\mathcal{O}\left(\varepsilon^{5}\right)
$$

where $S_{00}^{(c)}$ provides the force acting on the circular cylinder $r=$ $R ; S_{10}^{(c)}=$ linear function of the Fourier coefficients $f_{m}^{(c)}$ and $f_{m}^{(s)}$ in Eq. (22); and $S_{20}^{(c)}=$ quadratic function of these coefficients. A similar analysis is applied to calculations of the $y$-component of the force $\tilde{F}_{y}$.

Far from the cylinder $(r \gg R)$, Eq. (21) provides

$$
\begin{aligned}
& \phi_{n}(r, \theta) \sim \tilde{\phi}_{n}(\theta) \frac{e^{i k r}}{\sqrt{r}} \\
& \tilde{\phi}_{n}(\theta)=\sqrt{\frac{2}{\pi k}} e^{-i \frac{\pi}{4}}\left\{\frac{1}{2} \frac{G_{n 0}^{(c)}}{k H_{0}^{(1)^{\prime}}(k R)}+\sum_{m=1}^{\infty}\left[G_{n m}^{(c)} \cos (m \theta)+G_{n m}^{(s)} \sin (m \theta)\right] \frac{(-i)^{m}}{k H_{m}^{(1)^{\prime}}(k R)}\right\}
\end{aligned}
$$

The functions $\tilde{\phi}_{n}(\theta), n \geq 1$ depend on the Fourier coefficients $f_{\tilde{m}}^{(c)}, f_{m}^{(s)}, m \geq 0$ of the function $f(\theta)$. This dependence is linear for $\tilde{\phi}_{1}(\theta)$ and quadratic for $\tilde{\phi}_{2}(\theta)$. The obtained asymptotic formula for the diffracted wave field can be used to determine the shape of a vertical cylinder by using the wave measurements far from it.

Assume that the incident wave and the diffracted wave field (elevation of the water surface) far from a cylinder are known. Assume that the cylinder is vertical but the shape of its cross section and the position of the cylinder are not known. By analyzing the measured diffracted wave field and the derived first-order approximation of the potential

$$
\phi(r, \theta) \sim\left[\tilde{\phi}_{0}(\theta)+\varepsilon \tilde{\phi}_{1}(\theta)\right] \frac{e^{i k r}}{\sqrt{r}} \quad(r \rightarrow \infty)
$$

one can estimate the radius of the cylinder $(R)$, the position of its center, the scale of its surface perturbation $\varepsilon$, and the Fourier coefficients $f_{m}^{(c)}$ and $f_{m}^{(s)}$ of the deviation $f(\theta)$ of the cylinder cross section from the circular one. This problem of the cylinder identification is not considered in this paper. The algorithm of this study relates the function $\tilde{\phi}_{1}(\theta)$ and the shape function $f(\theta)$, which is crucial for efficient solution of the identification problem.

In some problems, the shape function can also depend on the small parameter $\varepsilon\{r=R[1+\varepsilon f(\theta, \varepsilon)]\}$ and can be approximated as

$f(\theta, \varepsilon)=f_{0}(\theta)+\varepsilon f_{1}(\theta)+\varepsilon^{2} f_{2}(\theta)+\varepsilon^{3} f_{3}(\theta)+\varepsilon^{4} f_{4}(\theta)+\mathcal{O}\left(\varepsilon^{5}\right)$

The asymptotic expansion [Eq. (29)], where each function $f_{n}(\theta)$ is presented by its Fourier series, is substituted in Eq. (16), and one again arrives at the boundary conditions in the form of Eq. (19) but with different functions $G_{n}(\theta)$. In particular, $f(\theta)$ is changed to $f_{0}(\theta)$ in Eq. (18). The expansion [Eq. (29)] is used in the next section to find an approximate solution of the problem for an elliptic cylinder with a small eccentricity. 


\section{Hydrodynamic Force on Elliptic Vertical Cylinder}

To validate the algorithm of the present paper, which is based on the asymptotic formula and operations with the Fourier coefficients, the algorithm is applied to the problem of elliptic vertical cylinders. This problem was solved by Chen and Mei (1971) by series of the Mathieu functions and elliptic coordinates, and by Williams (1985) using the method of integral equation on the boundary of the cylinder. Williams (1985) also used the asymptotic behaviors of the Mathieu functions to derive an asymptotic formula for the total force components when the eccentricity of the elliptic section is small.
In this section, the vertical cylinder with elliptic cross section of small eccentricity $e=\sqrt{1-b^{2} / a^{2}}$, where $a$ is the semimajor axis and $b$ is the semiminor axis of the elliptic cross section, is considered. The equation of the ellipse in the polar coordinates $(r, \theta)$ with the origin at the focus of the ellipse reads

$$
r=\frac{a\left(1-e^{2}\right)}{1-e \cos \theta}
$$

Taking the eccentricity $e$ as a small parameter of the problem $\varepsilon=e$ and calculating the Fourier coefficients of the right-hand side in Eq. (30), one obtains

$$
\begin{aligned}
r & =a \sqrt{1-\varepsilon^{2}}+2 a \sqrt{1-\varepsilon^{2}} \sum_{n=1}^{\infty}\left(\frac{\varepsilon}{1+\sqrt{1-\varepsilon^{2}}}\right)^{n} \cos (n \theta) \\
& =a+\varepsilon a \cos \theta+\varepsilon^{2} a\left[\frac{-1+\cos (2 \theta)}{2}\right]+\varepsilon^{3} a\left[\frac{-\cos \theta+\cos (3 \theta)}{4}\right]+\varepsilon^{4} a\left[\frac{-1+\cos (4 \theta)}{8}\right]+\mathcal{O}\left(\varepsilon^{5}\right)
\end{aligned}
$$

Comparing Eq. (31) with the equation of the cylinder in the present analysis $\{r=R[1+\varepsilon f(\theta, \varepsilon)]\}$ and expansion [Eq. (29)], one finds

$$
\begin{gathered}
R=a, f_{0}(\theta)=\cos \theta, f_{1}(\theta)=-\frac{1}{2}+\frac{1}{2} \cos (2 \theta) \\
f_{2}(\theta)=-\frac{1}{4} \cos \theta+\frac{1}{4} \cos (3 \theta), f_{3}(\theta)=-\frac{1}{8}+\frac{1}{8} \cos (4 \theta)
\end{gathered}
$$

The nondimensional force components $\tilde{F}_{x}$ and $\tilde{F}_{y}$ are calculated by the present algorithm using Eq. (26), Eq. (12) for the potential $\phi_{0}(R, \theta)$ on the cylinder at the leading order, and Eq. (21) for the higher-order potentials $\phi_{n}(R, \theta)$, where $n=1,2,3,4$. The modulus of the force components $\left|\tilde{F}_{x}\right|$ and $\left|\tilde{F}_{y}\right|$ is calculated by the fifth-order approximation [Eq. (15)] and by the third-order approximation, keeping only three terms in Eq. (15). The forces are calculated for $0<k a<4$ and the eccentricity $e=1 / 2$. The obtained forces are compared with the results by Williams (1985) in Figs. 2(a and b).

For this range of $k a$, it was found that the five terms in Eqs. (12) and (21) provide accurate results. At the end of each operation with the Fourier series, the resulting series was truncated to five terms with cosines and five terms with sines. Note that the forces were computed by Williams (1985) by two different methods, depending on the value of the product $k a$. For large $k a$, the forces were computed by the boundary element method, and for small $\mathrm{ka}$, the solution was found in the series form involving Mathieu functions. In the boundary element method, the elliptic contour was divided into 120 elements. Because of problems with convergence of the series of the Mathieu functions, Williams (1985) used the asymptotic formula of these functions for small eccentricity $e$ and obtained fifthorder approximations of the force components up to $\mathcal{O}\left(e^{5}\right)$ terms. The formulas for the coefficients in the asymptotic formula by Williams (1985) are collected in the four-page appendix at the end of that paper. The approach of the present paper is more straightforward and provides the approximations of the force components of the same order as by Williams (1985) for small eccentricity. The Mathieu functions in the infinite series solution depend on the parameter $q=(k a e)^{2} / 4$. Williams (1985) suggested using his asymptotic formula for $q \leq 0.4$ and the boundary element method for $q>0.4$. This implies that, in Figs. 2(a and b), the solid lines representing the forces by Williams (1985) for $e=1 / 2$ were computed by his asymptotic formula for $0<k a<2.5$. Figs. 2(a and b) show

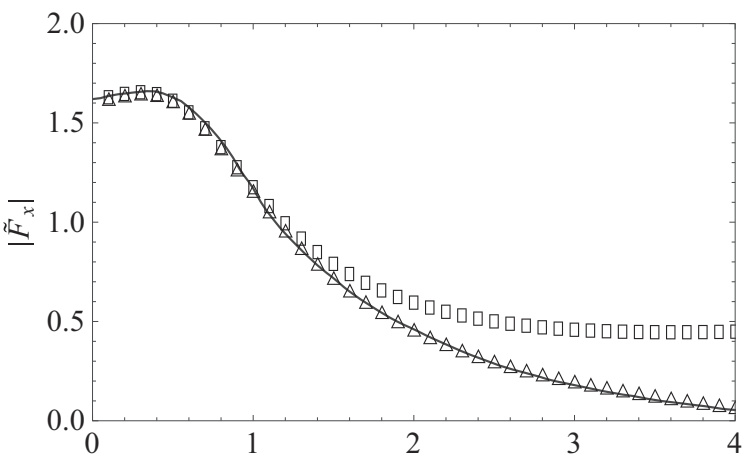

(a)

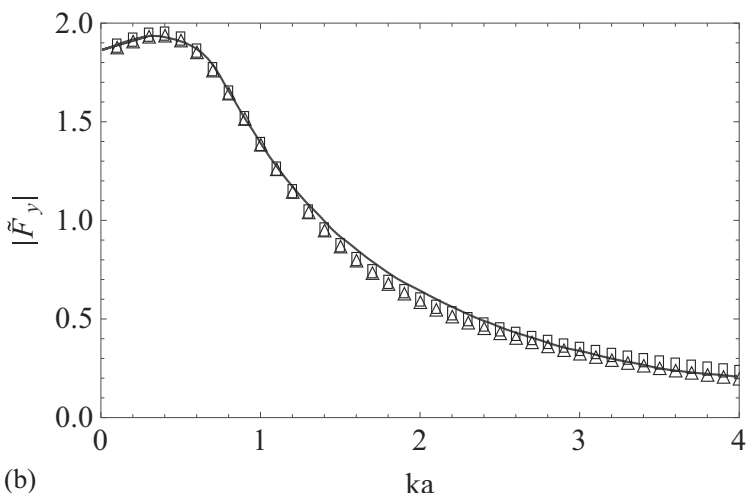

Fig. 2. The $x$ - and $y$-components of the nondimensional force acting on elliptic cylinder for $e=0.5$ : (a) $\alpha=0^{\circ}$; (b) $\alpha=90^{\circ}$ (Note: The solution by Williams (1985) is shown by the solid line, and the present asymptotic solutions of the third order and fifth order are shown by the square and triangle markers, respectively) 
that the present asymptotic fifth-order solutions is very close to the forces computed by Williams (1985). The present asymptotic solution in the long-wave approximation $(k a \rightarrow 0)$ provides

$$
\tilde{\mathcal{F}}_{x}(t)=\left[2-\frac{3}{2} \varepsilon^{2}-\frac{1}{8} \varepsilon^{4}+\mathcal{O}\left(\varepsilon^{6}\right)\right] \cos (\omega t)
$$

for $\alpha=0^{\circ}$ and

$$
\tilde{\mathcal{F}}_{y}(t)=\left[2-\frac{1}{2} \varepsilon^{2}-\frac{1}{8} \varepsilon^{4}+\mathcal{O}\left(\varepsilon^{6}\right)\right] \cos (\omega t)
$$

for $\alpha=90^{\circ}$. The asymptotic formulas [Eqs. (32) and (33)] coincide with those derived by Williams (1985).

\section{Hydrodynamic Force on Square Vertical Cylinder}

Let equation $r=a F(\theta)$ describe the square, $x= \pm a,-a<y<a$ and $y= \pm a,-a<x<a$, in the polar coordinates, $x=r \cos \theta$ and $y=r \sin \theta$. The Fourier coefficients of the function $F(\theta), 0 \leq$ $\theta \leq 2 \pi$, are determined, and then the corresponding Fourier series is converted into the form $r=R[1+\varepsilon f(\theta)]$, identifying values of $R, \varepsilon$, and the function $f(\theta)$. Then, the approach in the section "Vertical Cylinders with Nearly Circular Cross Sections" gives the components of the total hydrodynamic force acting on the square cylinder.

A square has four lines of symmetry:

$$
\begin{aligned}
F(-\theta) & =F(\theta), F\left(\frac{\pi}{2}-\theta\right)=F\left(\frac{\pi}{2}+\theta\right), F\left(\frac{\pi}{4}-\theta\right) \\
& =F\left(\frac{\pi}{4}+\theta\right)
\end{aligned}
$$

The Fourier series of the function $F(\theta)$ contains only $\cos (4 m \theta), m \geq 0$

$$
\begin{gathered}
F(\theta)=\frac{1}{2} F_{0}+\sum_{m=1}^{\infty} F_{m} \cos (4 m \theta) \\
F_{m}=\frac{8}{\pi} \int_{0}^{\pi / 4} F(\theta) \cos (4 m \theta) \mathrm{d} \theta=\frac{8}{\pi} \int_{0}^{\pi / 4} \frac{\cos (4 m \theta)}{\cos \theta} \mathrm{d} \theta
\end{gathered}
$$

where $F_{0}=(8 / \pi) \log (1+\sqrt{2}) ;$ and $F_{m}=(16 / \pi) \sqrt{2}(-1)^{m} /$ $[(4 m-1)(4 m-3)]+F_{m-1}, \quad m \geq 1$. Therefore, $R=a F_{0} / 2 \approx$ $1.1222 a$.

The maximum value of $F(\theta)$ is $\sqrt{2}$, which gives $\varepsilon=2 \sqrt{2}$ / $F_{0}-1 \approx 0.26$ and $|f(\theta)| \leqslant 1$, where

$$
f(\theta)=\sum_{m=1}^{\infty} f_{m} \cos (4 m \theta), \quad f_{m}=2 F_{m} /\left(\varepsilon F_{0}\right)
$$

where $f_{1}=-0.5357 ; f_{2}=0.1689 ; f_{3}=-0.0801 ; f_{4}=0.0463$; and $f_{5}=$ -0.03 . The shapes given by the equation $r=R[1+\varepsilon f(\theta)]$ with three terms (dashed line) and 10 terms (solid line) retained in the series [Eq. (34)] are shown in Fig. 3. It is seen that the approximation of the square cross section with only three terms in the series [Eq. (34)] is reasonably good.

Hydrodynamic forces acting on a square caisson have been studied by Mogridge and Jamieson (1976). They performed experiments with a $30.48 \times 30.48-\mathrm{cm}(12 \times 12-$ in. $)$ square

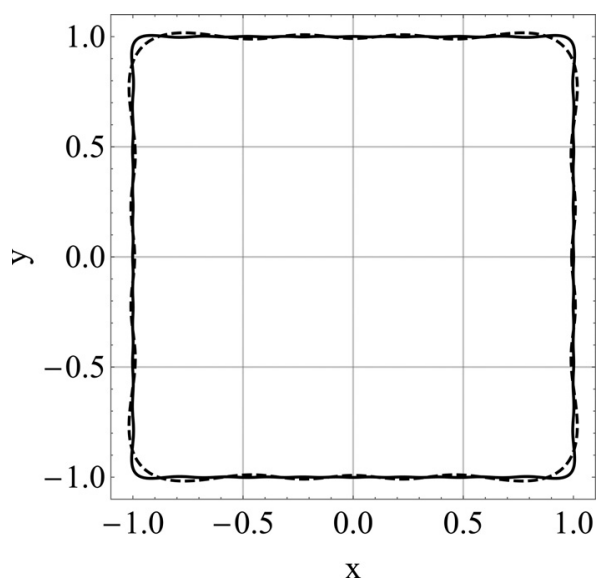

Fig. 3. Approximation of the square by the equation $r=R[1+\varepsilon f(\theta)]$ in the polar coordinates with three (dashed line) and 10 (solid line) terms retained in Eq. (34)

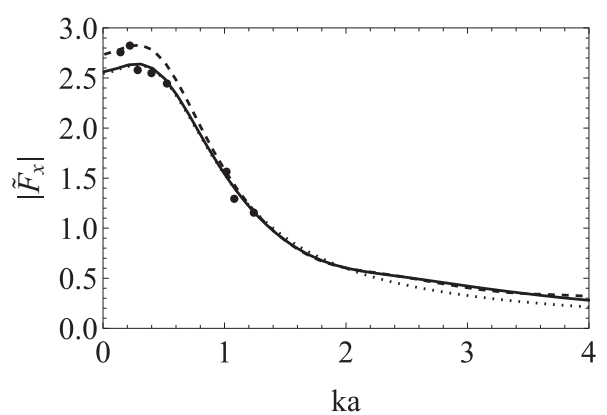

Fig. 4. Nondimensional hydrodynamic force acting on the square vertical cylinder in waves [Note: Comparison of Mogridge and Jamieson (1976) theory (dotted line), experimental results (filled circle markers), present method with one term in Eq. (34) (solid line), and present method with four terms in Eq. (34) (dashed line)]

box in a wave flume $3.65 \mathrm{~m}(12 \mathrm{ft})$ wide, $1.37 \mathrm{~m}(4.5 \mathrm{ft})$ deep, and $49.3 \mathrm{~m}$ (162 ft) long. The hydrodynamic forces for $\alpha=0^{\circ}$ were measured and compared with the predictions by the theory of equivalent circular radius. In this theory of equivalent circular radius, the horizontal hydrodynamic force on a vertical cylinder with the area of its cross section $|D|$ is approximated by the force acting on the circular cylinder of radius $R_{e}$, where the area of the circular cross section $\pi R_{e}^{2}$ is equal to the area $|D|$. For the square-shape vertical cylinder with $|D|=(2 a)^{2}$, one obtains $\mathrm{R}_{\mathrm{e}}=2 a / \sqrt{\pi} \approx 1.1283 a$. It is seen that the equivalent radius $\mathrm{R}_{\mathrm{e}}$ is very close to the radius $R \approx 1.1222 a$ calculated earlier by using the Fourier series.

The computed hydrodynamic forces acting on the square vertical cylinder with $\alpha=0^{\circ}$ are shown in Fig. 4. The fifth-order approximation [Eq. (15)] was used. Note that $\varepsilon^{5}<0.0012$. Representing the square with four terms in Eq. (34) and truncating the Fourier series of the potentials $\phi_{n}, n=0,1,2,3,4$, in Eq. (21) to 16 terms, the force shown by the dashed line is obtained. The computed force is very close to the experimental results by Mogridge and Jamieson (1976), which are shown by markers. Keeping just one term in Eq. (34) and four terms in the Fourier series [Eq. (21)], one arrives at the solid line, which is very close to the prediction of the force with four terms in Eq. (34), where 
$1 \leq k a \leq 4$, but underpredicts the force in the interval $0 \leq k a \leq 1$. The force predicted by the equivalent circular radius theory of Mogridge and Jamieson (1976) is shown by the dotted line in Fig. 4. This prediction of the force is very close to the present asymptotic force with one term in Eq. (34), where $0 \leq k a<2$. The authors conclude that more terms in Eq. (34) provide a better approximation of the experimental force for long waves but do not improve the prediction of the hydrodynamic force for short waves.

\section{Hydrodynamic Force on Quasi-Elliptic Vertical Cylinder}

A quasi-ellipse consists of a rectangular part in the center and two semicircular parts at the front and back [Fig. 5(a)]. In Fig. 5(a), $D / 2$ is the radius of the semicircles, and $B$ is the length of the rectangular part.

Let $r=F(\theta)$ describe the quasi-ellipse in Fig. 5(a) in the polar coordinates, $x=r \cos \theta$ and $y=r \sin \theta$, where

$$
\begin{aligned}
& 0 \leq \theta \leq \arctan \frac{D}{B} \\
& \arctan \frac{D}{B} \leq \theta \leq \pi-\arctan \frac{D}{B} \\
& \pi-\arctan \frac{D}{B} \leq \theta \leq \pi+\arctan \frac{D}{B} \\
& \pi+\arctan \frac{D}{B} \leq \theta \leq 2 \pi-\arctan \frac{D}{B} \\
& 2 \pi-\arctan \frac{D}{B} \leq \theta \leq 2 \pi
\end{aligned}
$$

Eq. (35) are shown in Fig. 5(b). It is seen that the approximation for the quasi-elliptic cross section with eight terms in the series Eq. (35) is reasonably good.
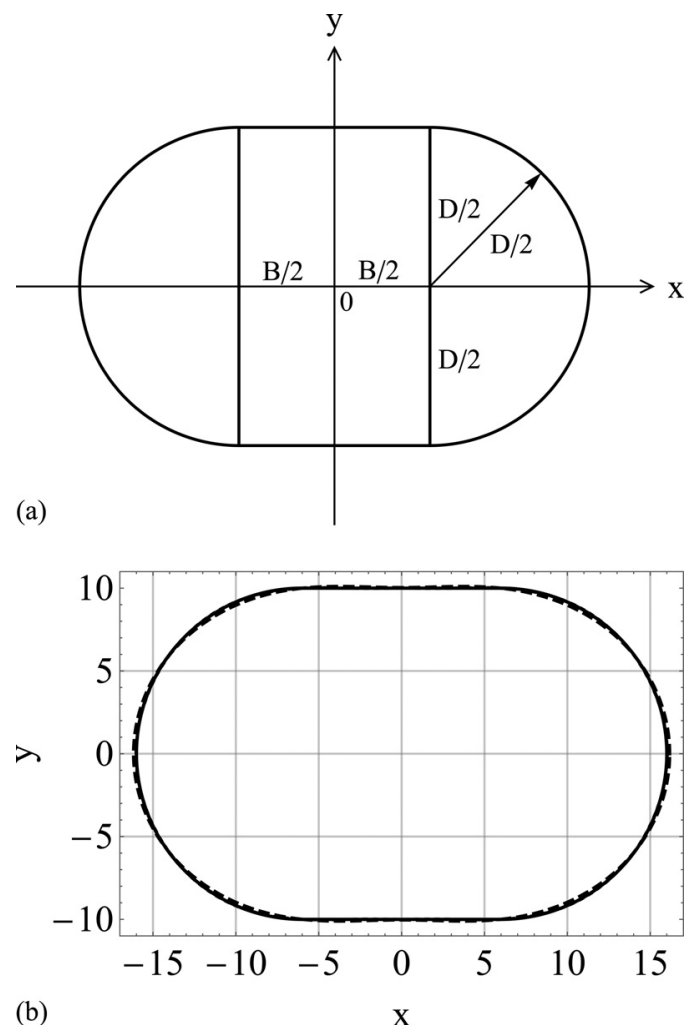

Fig. 5. (a) Quasi-ellipse; (b) approximation of quasi-ellipse by the equation $r=R[1+\varepsilon f(\theta)]$ in the polar coordinates with two (dashed line) and eight (dotted line) terms retained in Eq. (35) and the exact shape of quasi-ellipse (solid line) where $f_{1}=3.06712 ; f_{2}=-0.145175 ; f_{3}=-0.0595601 ; f_{4}=0.050526$ $f_{5}=-0.0148836 ; f_{6}=-0.00652338 ;$ and $f_{7}=-0.00973661$. The shapes given by the equation $r=R[1+\varepsilon f(\theta)]$ with two terms (dashed line) and eight terms (solid line) retained in the series 
Wang et al. (2011) developed a three-dimensional time domain method to solve the Navier-Stokes equations including viscosity and nonlinear effects. Wave forces on the quasi-ellipse caisson were calculated and compared with the results of Wang et al. (2011) (Fig. 6). For comparison purposes, the vertical axis in Fig. 6 is chosen as $F_{x}^{*}=(\pi k a / 4) \tilde{F}_{x}, a=(B+D) / 2=0.8 D$. Fig. 6 shows that there is a small discrepancy between the present results and the results of Wang et al. (2011), which can be attributed to the effect of viscosity and nonlinearity considered in the Navier-Stokes formulation of Wang et al. (2011). Fig. 6 shows that the present approach gives slightly smaller values for wave forces compared with the Navier-Stokes computations. However, the present model can still be used to make a quick study to identify critical wave numbers and directions as input to more detailed and computationally more expensive Navier-Stokes computations.

For the incident wave propagating at an angle $\alpha$ to the positive $x$-axis, the wave force and the maximum wave run-up are computed

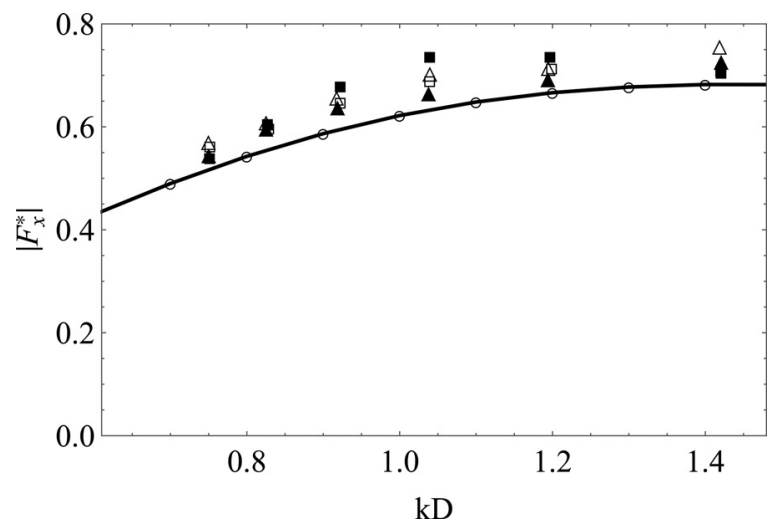

Fig. 6. Nondimensional hydrodynamic force acting on the quasi-elliptic vertical cylinder in waves [Note: Wang et al. (2011) for $H / h=0.05$ (filled squares), $H / h=0.095$ (empty squares), $H / h=0.15$ (filled triangles), and $H / h=0.2$ (empty triangles), and the present method with 14 terms in Eq. (35) (line with empty circle markers); here, $H$ is the wave height $H=2 A$, and $h$ is the water depth] and compared for cylinders of circular, elliptic, quasi-elliptic, and square cross sections (Fig. 7) with the same cross-sectional area. The forces and maximum run-ups for $\alpha=0^{\circ}$ are compared in Figs. 8(a and b), respectively. Fig. 8(a) shows that the wave force is smallest for the cylinder with a quasi-elliptic cross section, and Fig. 8(b) shows that maximum nondimensional wave run-up is highest for the square cylinder. For the incident wave propagating at angle $\alpha=90^{\circ}$ to the positive $x$-axis (Fig. 7), wave forces for elliptic and quasi-elliptic cylinders are higher than for $\alpha=0^{\circ}$. This can be attributed to the larger projected area normal to the flow of these cylinders for $\alpha=90^{\circ}$. The computations for $\alpha=90^{\circ}$ provide that the wave force is highest for the cylinder with a quasi-elliptic cross section, and the maximum nondimensional wave run-up is highest for the quasi-elliptic cylinder, where $0<k a<1.1$, and for the square cylinder, where $1.1<k a<4$. The figures for the latter case is not included. It is concluded that the forces are dependent on angle of wave incidence $\alpha$ with the corresponding corrections of order $O(\varepsilon)$ [see Eq. (26), where $S_{00}^{(c)}$ is independent of $\alpha$ ].

\section{Hydrodynamic Force and Wave Run-up on Cylinder with Cosine-Type Radial Perturbations}

Mansour et al. (2002) studied vertical cylinders with cosine-type radial perturbations of their cross section

$$
r=R[1+\varepsilon \cos (N \theta)]
$$

where $N=$ positive integer. The cross sections of the cylinders in Eq. (36) are shown in Fig. 9 for $R=1 \mathrm{~m}, \varepsilon=0.05$, and $N=1,2,3,4,5,6$.

Mansour et al. (2002) derived the leading-order corrections to the forces acting on the cylinders Eq. (36) as $\varepsilon \rightarrow 0$, and to the maximum nondimensional run-up $\triangle_{\max } / 2 A$ for these cylinders. They also computed the hydrodynamic forces and wave run-ups by the method of boundary integral equation and compared their numerical and first-order asymptotic results. They concluded that the first-order asymptotic solutions agree well with the numerical solutions in the range $0 \leq \varepsilon \leq 0.05$. The higher-order asymptotic solution of the

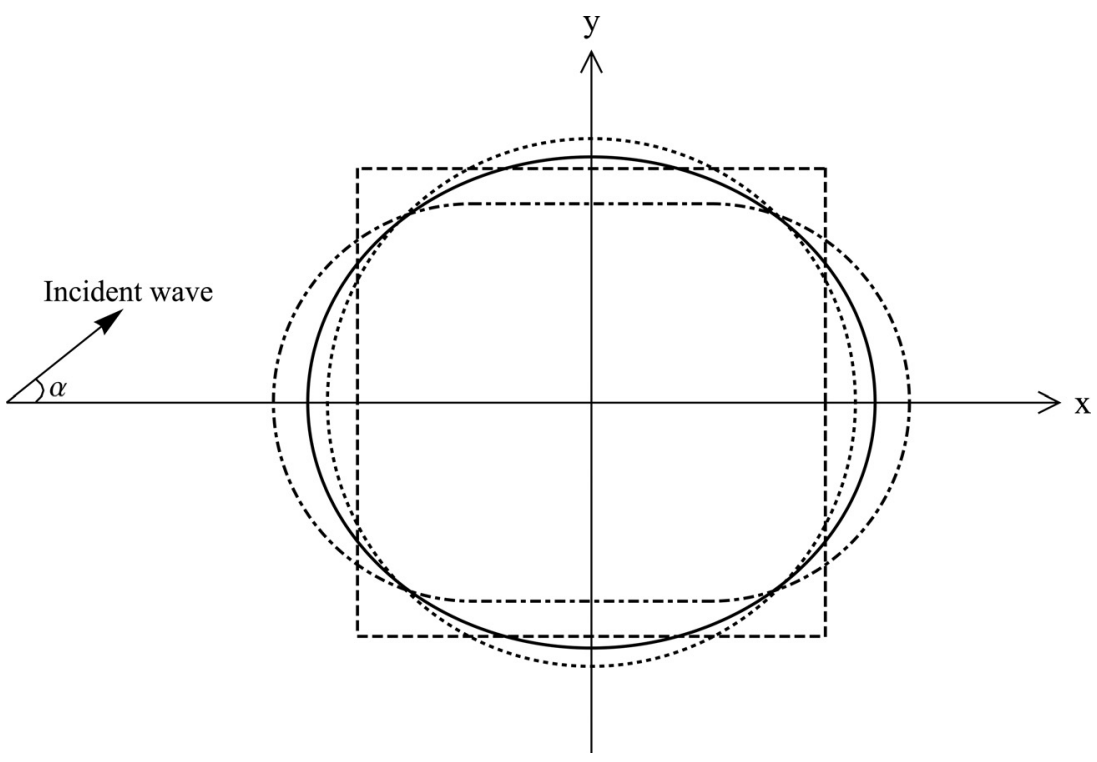

Fig. 7. Orientation of quasi-elliptic (dot-dashed line), elliptic (solid line), square (dashed line), and circular (dotted line) cylinders relative to incident wave 


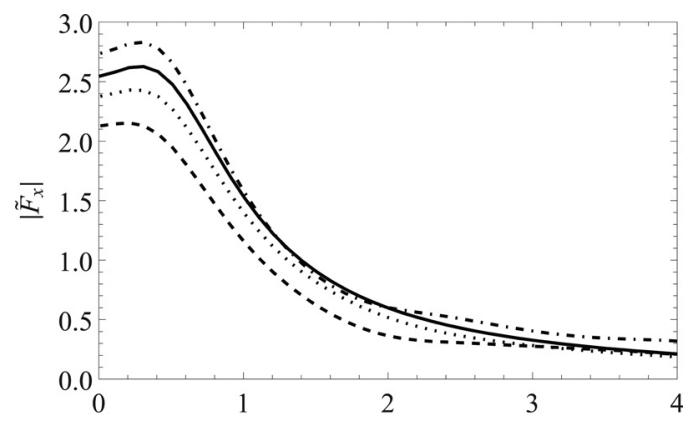

(a)

$\mathrm{ka}$

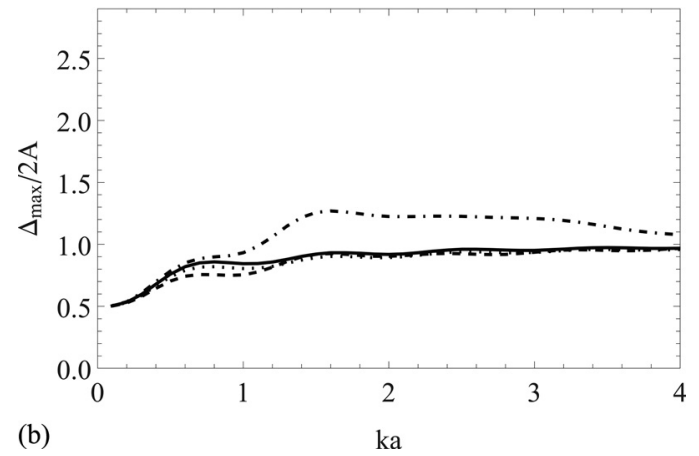

Fig. 8. (a) Nondimensional hydrodynamic force and (b) maximum nondimensional wave run-up on cylinders of circular (solid line), elliptical (dotted line), quasi-elliptical (dashed line), and square (dot-dashed line) cross sections with same cross-sectional areas [Note: Angle of wave incidence $\alpha$ is zero]

present paper provides forces and run-ups almost identical with the numerical forces and run-ups by Mansour et al. (2002) (Figs. 10 and 11). It is observed that the present approach compares quite well with the integral equation method of Mansour et al. (2002) even for high values of $k R$. Note that the asymptotic results by Mansour et al. (2002) for the maximum nondimensional wave run-up deviate significantly from their numerical results for $k R \geq 1$.

To compare the forces computed by Mansour et al. (2002) with the results of the present method, the forces in Eqs. (7) and (8) are multiplied by $(1 / 2) \tanh (k h)$. The resulting nondimensional forces $\left|\bar{F}_{x}\right|=[(1 / 2) \tanh (k h)]\left|\vec{F}_{x}\right|$ are shown in Figs. 10(a-d) for $N=2,3$, 4, and 6 in Eq. (36), Figs. 10(a-d) demonstrate that the first-order asymptotic forces by Mansour et al. (2002) are very close to both the numerical results and the present higher-order forces for $\varepsilon=$ 0.05 and $0<k R<4$. The maximum run-up $\triangle_{\max } /(2 A)$ is more sensitive to the number of terms in the asymptotic solution [Eq. (15)] [Figs. 10(e-h)]. It is seen that the present fifth-order asymptotic solution provides a wave run-up almost identical to the numerical solution for $\varepsilon=0.05$. This conclusion is also true for $\varepsilon=0.1$ (Fig. 11) for both the force and the maximum run-up as functions of the nondimensional wave number $k R$. Note that the first-order asymptotic solution cannot be used for $\varepsilon=0.1$.

The present method is restricted to vertical cylinders whose cross sections are close to a circle. Liu et al. (2016) numerically solved Eqs. (2)-(4) with no restriction on the shape of the cylinder cross section. The method they used is not an asymptotic method but a Fourier series method combined with the Galerkin method to satisfy the body condition [Eq. (4)]. However, the authors reported some difficulties with the system of equations they obtained. The system is ill posed for some cases after truncating the infinite system of equations. Despite the reported difficulties, their results show
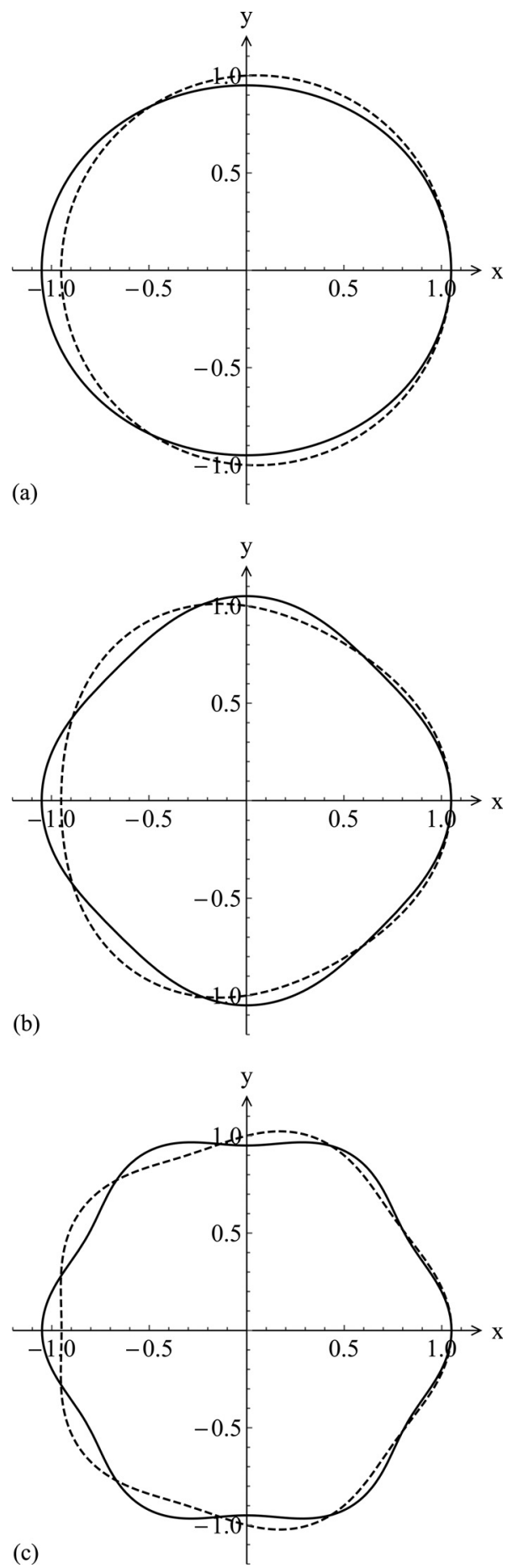

Fig. 9. Cross sections of the cylinders [Eq. (36)] for $R=1 \mathrm{~m}$, $\varepsilon=0.05$, and (a) $N=1$ (dashed line), $N=2$ (solid line), (b) $N=3$ (dashed line), $N=4$ (solid line), (c) $N=5$ (dashed line), $N=6$ (solid line)

good agreement with numerical results by Mansour et al. (2002). The present method deals only with multiplication and summation of Fourier series to find the unknown potentials $\phi_{n}, n=1,2,3,4$, so the present method is stable in solving the wave diffraction problem for vertical cylinders.

The effect of the truncation of the Fourier series Eqs. (12), (21), and (22) on the performance of the present asymptotic solution is demonstrated by Figs. 12 and 13. Let the number of terms $m$ vary from 1 to $p$ in Eqs. (21) and (22). After each multiplication of two Fourier series, the resulting Fourier series is truncated to $p$ terms. 

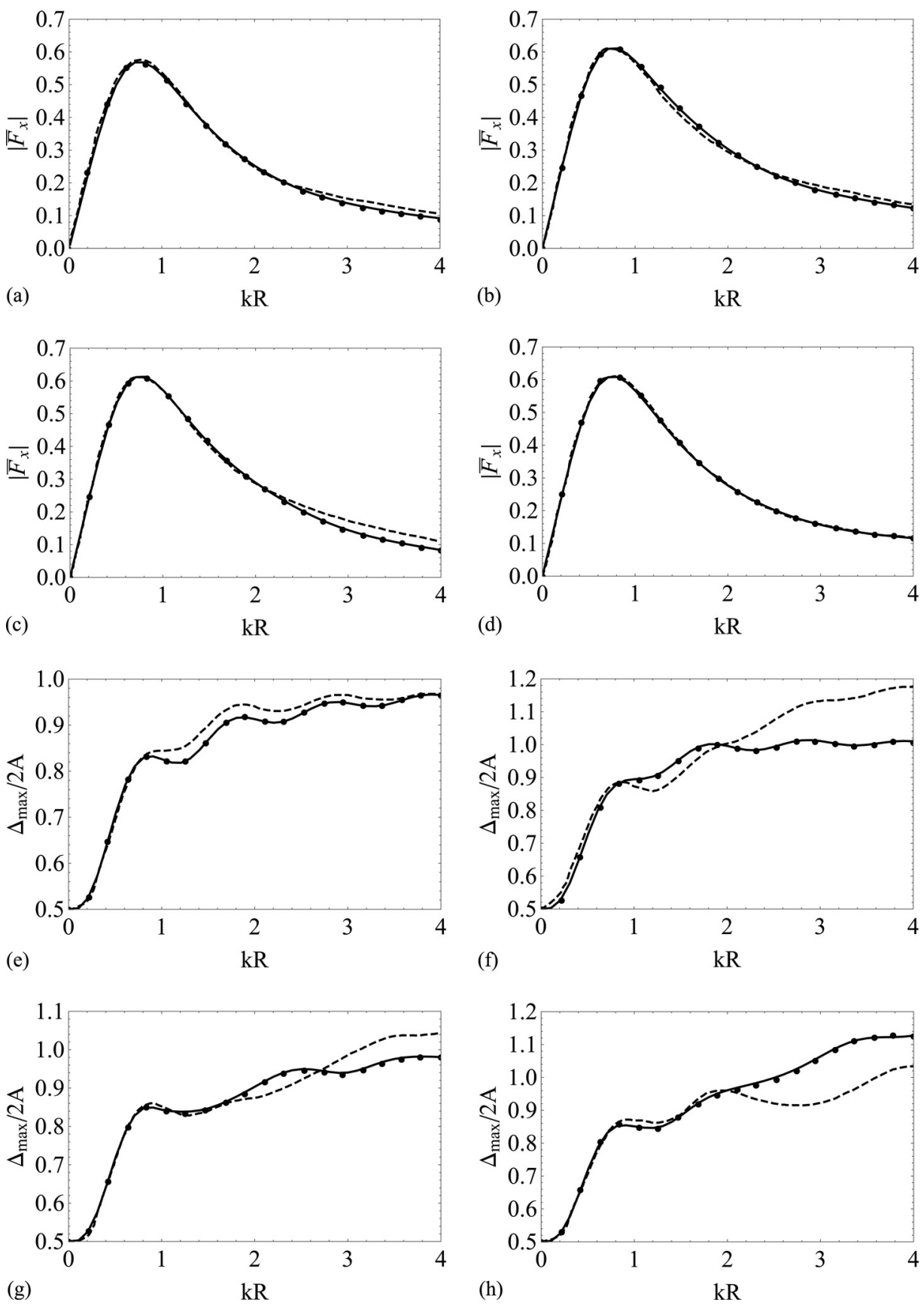

Fig. 10. (a-d) Nondimensional hydrodynamic force and (e-h) maximum nondimensional wave run-up on the vertical cylinder in Eq. (36); comparison of results by the present method (solid line) with the analytical (dashed line) and numerical (filled circles) results by Mansour et al. (2002) for $\varepsilon=$ 0.05 and (e) $N=2$ in (a) and (e); $N=3$ in (b) and (f); $N=4$ in (c) and (g), and $N=6$ in (d) and (h)

The system of the equation in the method of Liu et al. (2016) is truncated in a similar way because Fourier series are used to represent the body shape and the velocity potential.

Liu et al. (2016) recommended $p=20$ for any shapes of the cylinders with cosine-type section. It was observed that $p=12$ in the present asymptotic solution provides good agreement with the numerical results by Liu et al. (2016). Even $p=6$ in the present method provides a very reasonable agreement with the numerical force and maximum run-up. The distributions of the wave run-up along the cylinder are shown in Fig. 13 for $p=20$ in the computations by Liu et al. (2016) and $p=6,12$ in the present calculations. The predictions of the wave run-up by the present method are good even for waves with $k R=4$.

\section{Long-Wave Approximation of Wave Forces}

The formula [Eq. (5)] for the hydrodynamic force $\mathbf{F}(t)$ acting on a vertical cylinder with cross section $D$ can be simplified for long waves, where $k a \rightarrow 0$ and $a$ is a characteristic dimension of the cylinder cross section. The ideas by Haskind (1973, Chapter 2) are used here, who expressed the forces as integrals of the potential of the incident wave, three radiation potentials, and their normal derivatives on the cylinder. The radiation potentials describe waves generated by the cylinder oscillating in $x$ - and $y$-directions and due to torsional oscillation of the cylinder. Haskind (1973) also introduced generalized added masses and damping coefficients of vertical cylinders as functions of the nondimensional wave number $k a$. The generalized added masses 


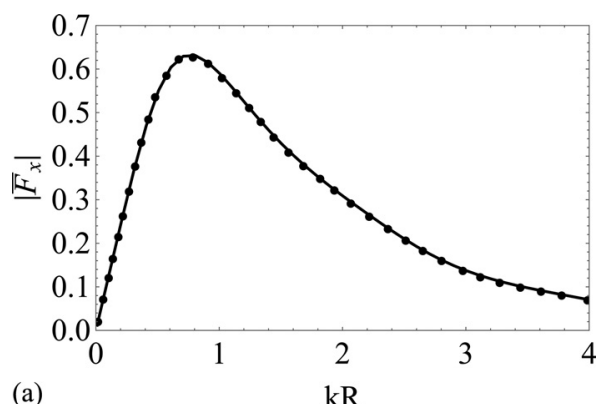

(a)

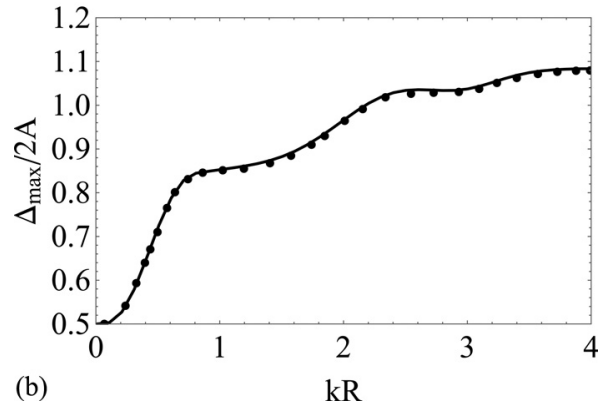

Fig. 11. (a) Nondimensional hydrodynamic force and (b) maximum nondimensional wave run-up on the vertical cylinder in Eq. (36); comparison of the present method (solid line) with the numerical method of Mansour et al. (2002) (filled circles) for $\varepsilon=0.1$ and $N=4$

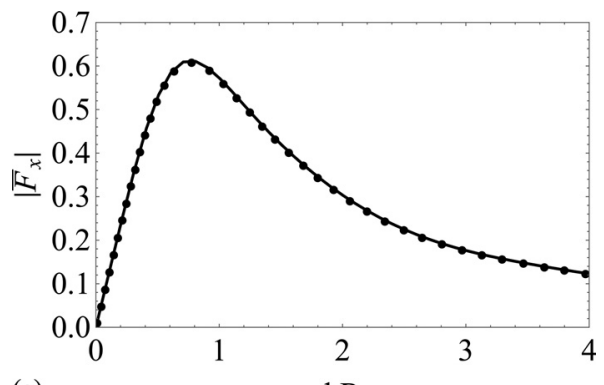

(a)

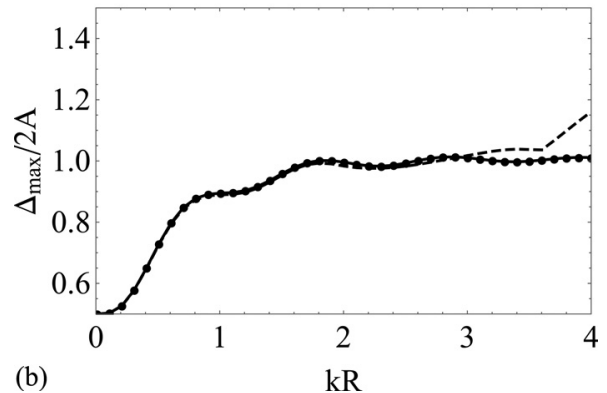

Fig. 12. Effect of truncation number $p$ on the nondimensional (a) hydrodynamic force and (b) maximum wave run-up for $\varepsilon=0.05$ and $N=3$ in Eq. (36), Liu et al. (2016) numerical method with $p=9,20$ (filled circles), and the present method with $p=4$ (dashed line) and $p=9$ (solid line)

approach the added masses of the two-dimensional body $D$ moving in unbounded incompressible liquid as $k a \rightarrow 0$. This section is limited to the force component in the direction of the incident wave propagation in the limit as $k a \rightarrow 0$, with $\alpha=0^{\circ}$.

Eqs. (5) and (6) provide the nondimensional $x$-component of the hydrodynamic force acting on the cylinder

$$
\tilde{F}_{x}=\frac{-i}{\pi a^{2} k} \int_{\partial D} \phi(r, \theta) n_{x} d s
$$

where $\phi(r, \theta)=$ solution of Eqs. (2)-(4); and $n_{x}=x$-component of the unit normal vector $\mathbf{n}$ to the surface of the cylinder. It is convenient to introduce new potential $\varphi(x, y)$ by the equation $\phi(r, \theta)=e^{i k x}-i k \varphi(x, y)$. The potential $\varphi(x, y)$ satisfies Eq. (2) and describes outgoing waves as $r \rightarrow \infty$, and its normal derivative on the cylinder is given by

$$
\frac{\partial \varphi}{\partial n}=e^{i k x} n_{x} \quad(\text { on } \partial D)
$$

By using the potential $\varphi(x, y)$ and Eq. (38), the force [Eq. (37)] can be presented in the form

$$
\tilde{F}_{x}=\frac{1}{\pi a^{2}}\left(-\frac{i}{k} \int_{\partial D} e^{i k x} n_{x} d s-\int_{\partial D} \varphi \frac{\partial \varphi}{\partial n} e^{-i k x} d s\right)
$$

The product $e^{i k x} n_{x}$ in the first integral of Eq. (39) can be viewed as the scalar product of two vectors: $\left(e^{i k x}, 0\right)$ and $\mathbf{n}$. Then, the divergence theorem yields

$$
-\frac{i}{k} \int_{\partial D} e^{i k x} n_{x} d s=-\frac{i}{k} \int_{D} \operatorname{div}\left(e^{i k x}, 0\right) d x d y=\int_{D} e^{i k x} d x d y
$$

Taking the limit in Eq. (39) as $k a \rightarrow 0$, where $x / a=\mathcal{O}(1)$, one obtains

$$
\tilde{F}_{x}(0)=\frac{1}{\pi a^{2}}\left(|D|-\int_{\partial D} \varphi_{0} \frac{\partial \varphi_{0}}{\partial n} d s\right)
$$

where $|D|=$ area of the cylinder cross section; and $\varphi_{0}(x, y)=$ limiting value of the potential $\varphi(x, y)$ as $k a \rightarrow 0$. The potential $\varphi_{0}(x, y)$ satisfies the following equations:

$$
\begin{gathered}
\nabla^{2} \varphi_{0}=0 \quad(\text { outside } D) \\
\frac{\partial \varphi_{0}}{\partial n}=n_{x} \quad(\text { on } \partial D) \\
\varphi \rightarrow 0 \quad\left(x^{2}+y^{2} \rightarrow \infty\right)
\end{gathered}
$$

and describes the two-dimensional flow caused by the motion of the body $D$ in unbounded and incompressible liquid in the $x$-direction at the unit speed. The integral in Eq. (40) multiplied by $-\rho$ is known as the added mass $m_{x x}$. Finally

$$
\tilde{F}_{x}(0)=\frac{1}{\pi a^{2}}\left(|D|+\frac{m_{x x}}{\rho}\right)
$$

For the elliptic cylinder $x^{2} / a^{2}+y^{2} / b^{2}=1$ with the semimajor axis $a$ and the semiminor axis $b$, where $b=a \sqrt{1-e^{2}}$ and $e$ is the eccentricity of the ellipse, $|D|=\pi a b, m_{x x}=\rho \pi b^{2}$ and Eq. (41) gives

$$
\tilde{F}_{x}(0)=\frac{b}{a}+\frac{b^{2}}{a^{2}}=\sqrt{1-e^{2}}+1-e^{2}=2-\frac{3}{2} e^{2}-\frac{1}{8} e^{4}+\mathcal{O}\left(e^{6}\right)
$$

which corresponds to the asymptotic formula Eq. (32). For the elliptic cylinder $x^{2} / b^{2}+y^{2} / a^{2}=1,|D|=\pi a b, m_{x x}=\rho \pi a^{2}$ and Eq. (41) gives

$$
\tilde{F}_{x}(0)=\frac{b}{a}+1=2-\frac{1}{2} e^{2}-\frac{1}{8} e^{4}+\mathcal{O}\left(e^{6}\right)
$$



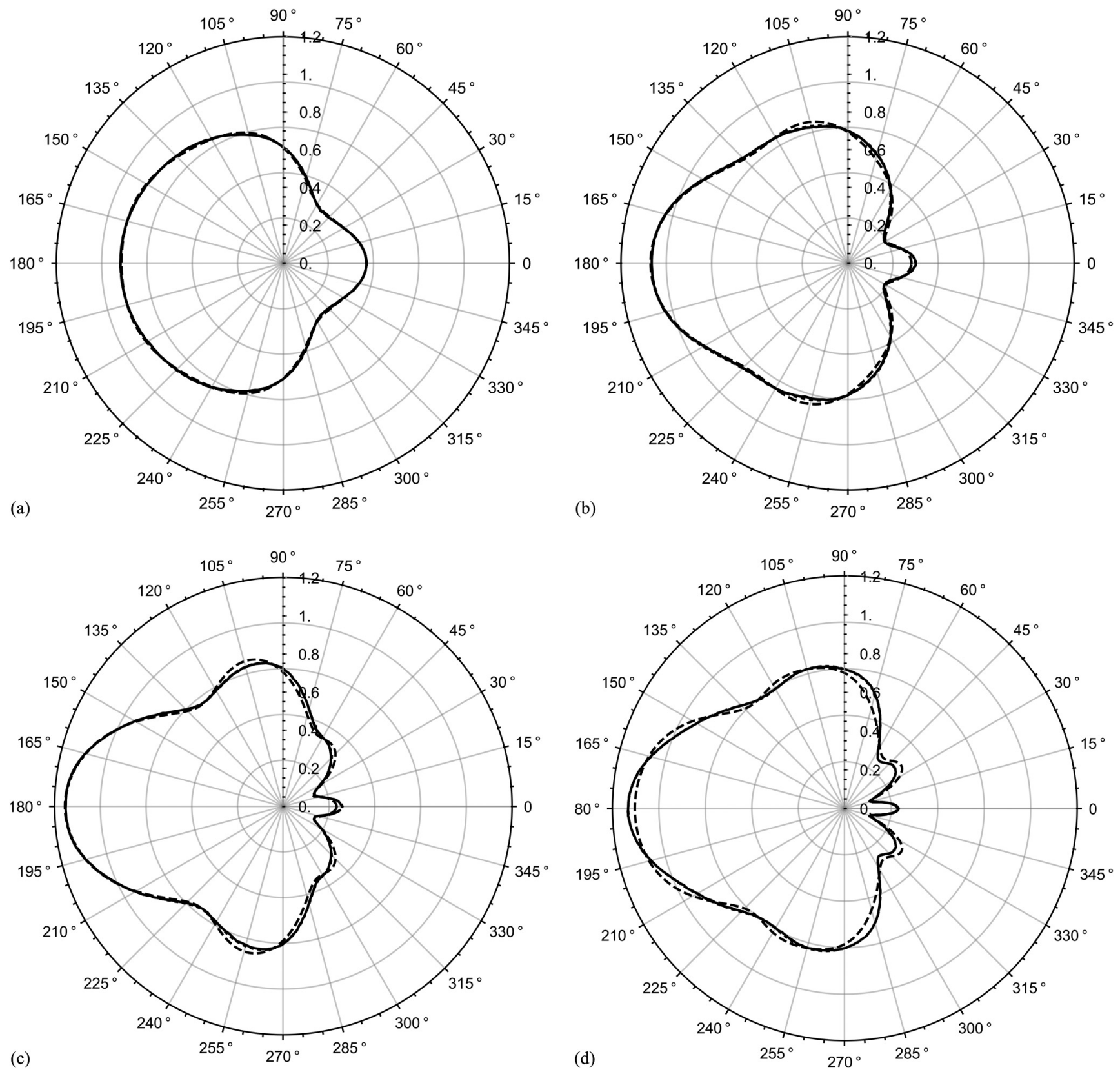

Fig. 13. Nondimensional wave run-up $\triangle(\theta) / 2 A$ for the cylinder [Eq. (36)] with $\varepsilon=0.05$ and $N=5$ computed for (a) $k R=1$, (b) $k R=2$, (c) $k R=3$, and (d) $k R=4$; present asymptotic method with $p=6$ (dashed line) and $p=12$ (dotted line) is compared with the numerical results (solid line) by Liu et al. (2016) with $p=20$

which corresponds to the asymptotic formula Eq. (33).

For the square cylinder with side $2 a,|D|=4 a^{2}$ and $m_{x x} \approx$ $1.51 \rho \pi a^{2}$, which gives $\tilde{F}_{x}(0) \approx(4 / \pi)+1.51 \approx 2.7832$.

The fifth-order approximation from the section "Hydrodynamic Force on Square Vertical Cylinder" with four terms in Eq. (34) gives $\left|\tilde{F}_{x}(0)\right| \approx 2.736$, and just one term in Eq. (34) gives $\left|\tilde{F}_{x}(0)\right| \approx 2.563$. The latter value is close to that predicted by the theory of equivalent circular radius (Fig. 4). Therefore, the theory by Mogridge and Jamieson (1976) underpredicts the force for long waves by approximately $8.5 \%$.

\section{Conclusion}

An asymptotic approach to the linear problem of regular water waves interacting with a vertical cylinder with an arbitrary cross section was presented. The incident regular wave is onedimensional, water is of finite depth, and the rigid cylinder extends from the bottom to the water surface. Deviation of the cylinder surface from a mean circular cylinder is assumed small compared with the radius of the mean circular cylinder. The fifth-order asymptotic solution of the problem was obtained. Each term in the asymptotic expansion of the velocity potential is the solution of a radiation problem for the circular cylinder. These radiation problems differ only by the value of the normal derivative of the corresponding potential on the surface of the circular cylinder. The radiation problems were solved by the Fourier method. The numerical solution of the problem was reduced to operations with the Fourier coefficients of the potentials and the shape function. The numerical algorithm was applied to the problems of wave diffraction by 
elliptic, square, and quasi-elliptic cylinders and by the cylinder with cosine-type radial perturbation. The obtained results were compared with experimental and numerical results by others in terms of the hydrodynamic forces and wave run-up on cylinders in waves. The present approach provides forces very close to those computed numerically and measured in experiments for relatively long incident waves, $0<k R<4$, where $2 \pi / k$ is the wave length of the incident wave. The present approach should be used with care for short incident waves. A reason for this conclusion comes from the body boundary condition. The analysis of the velocity potentials $\left(\phi_{n}\right)$ revealed that they have terms with factors $(k R)^{2 n}$. Correspondingly, the expansion [Eq. (15)] is formally asymptotic only if $\varepsilon(k R)^{2} \ll 1$. For small values of $\varepsilon$ and short waves with $\lambda / R=\mathcal{O}\left(\varepsilon^{1 / 2}\right)$, the method of renormalization or the multiscale method can be used to derive uniformly valid asymptotic expansions of the hydrodynamic forces.

The asymptotic method of this paper was validated for the longwave approximation. The long-wave approximation provides the forces acting on a vertical cylinder with an arbitrary cross section in linear regular waves through the area of the cylinder cross section and the added masses of this cross section. The values of the forces at $k R=0$ are exact within the linear wave theory. The added mass tables can be used to calculate the forces at $k R=0$.

An advantage of the present approach compared with the numerical solution of the problem by a boundary element method is that it provides the forces and the diffracted wave field in terms of the Fourier series of the deviation of the cylinder shape from the circular one. The leading-order potential $\phi_{0}(r, \theta)$ is independent of these coefficients, the first-order potential $\phi_{1}(r, \theta)$ is a linear form of these coefficients, and the second-order potential $\phi_{2}(r, \theta)$ is a quadratic form of the coefficients. By using these forms of the potentials $\phi_{n}(r, \theta)$, one can formulate the problem of identification of the cylinder and its shape with the help of measured elevations of water surface far from the cylinder. One can also determine how much some small variations of a cylinder shape change the loads acting on this cylinder in waves, and optimize the shape of the cylinder to approach certain restrictions on the loads.

It was found that cylinders with quasi-elliptic cross sections experience the least wave force compared with cylinders of elliptic, square, and circular cylinders with the same cross-sectional area for zero angle of wave incidence (Fig. 7).

In real applications, cylinders are always arranged in groups; therefore, the analysis of the so-called hydrodynamic interaction problem of several noncircular cylinders in waves should be carried out. The present method is very suitable for analysis of this problem, and the authors suggest solving the problem by iterations satisfying the boundary condition on each cylinder one after another and using the addition theorem of the Bessel functions. The iterations are suggested to combine with the asymptotic approach of the present paper to improve the convergence of the iterations.

\section{Acknowledgments}

Preliminary results of this paper were reported at the International Workshop on Water Waves and Floating Bodies 2015 (Disibuyuk and Korobkin 2015). This research was started while the first author was visiting the School of Mathematics, University of East Anglia, as a visiting research scientist during the period from October 2014 to February 2015. This visit was supported by YÖK (Council of Higher Education of Turkey). The first author was also supported by a grant (BIDEB-2211) from TÜBITAK (Scientific and Technological Research Council of Turkey). These supports are greatly acknowledged. The authors thank the reviewers for their suggestions.

\section{References}

Au, M. C., and Brebbia, C. A. (1983). "Diffraction of water waves for vertical cylinders using boundary elements." Appl. Math. Modell., 7(2), 106-114.

Black, J. L., Mei, C. C., and Bray, M. C. G. (1971). "Radiation and scattering of water waves by rigid bodies." J. Fluid Mech., 46(1), 151-164.

Chen, H. S., and Mei, C. C. (1971). "Scattering and radiation of gravity waves by an elliptical cylinder." Rep. No. 140, Parsons Laboratory, Dept. of Civil Engineering, Massachusetts Institute of Technology, Cambridge, MA.

Chen, H. S., and Mei, C. C. (1973). "Wave forces on a stationary platform of elliptical shape.” J. Ship Res., 17(2), 61-71.

De Vos, L., Frigaard, P., and De Rouck, J. (2007). "Wave run-up on cylindrical and cone shaped foundations for offshore wind turbines." Coastal Eng., 54(1), 17-29.

Disibuyuk, N. B., and Korobkin, A. A. (2015). "Wave forces on a vertical cylinder with non-circular cross section." Proc., 30th Int. Workshop On Water Waves And Floating Bodies, International Workshop on Water Waves and Floating Bodies, Oslo, Norway, 53-56.

Eatock Taylor, R., and Hu, C. S. (1991). "Multipole expansions for wave diffraction and radiation in deep water." Ocean Eng., 18(3), 191-224.

Eatock Taylor, R., and Hung, S. M. (1987). "Second order diffraction forces on a vertical cylinder in regular waves." Appl. Ocean Res., 9(1), 19-30.

Fichtenholtz, G. M. (2001). Course of differential and integral calculus, Vol. 3, Fizmatlit, Moscow (in Russian).

Garrett, C. J. R. (1971). "Wave forces on a circular dock." J. Fluid Mech., 46(1), 129-139.

Haskind, M. D. (1973). Hydrodynamic theory of ship rolling, Fizmatlit, Moscow (in Russian).

Havelock, T. H. (1940). "The pressure of water waves upon a fixed obstacle.” Proc. R. Soc. A, 175(963), 409-421.

Hwang, L.-S., and Tuck, E. O. (1970). "On the oscillations of harbours of arbitrary shape.” J. Fluid Mech., 42(3), 447-464.

Iafrati, A., and Korobkin, A. A. (2006). "Breaking wave impact onto elastic wall." Proc., 4th Int. Conf. on Hydroelasticity in Marine Technology, National Defence Industry Press, Beijing, 139-148.

Isaacson, M. (1978). "Vertical cylinders of arbitrary section in waves." $J$. Waterway, Port, Coastal, Ocean Div., 104(3), 309-324.

Korobkin, A. A. (2008). "Wagner theory of steep wave impact." Proc., $23 r d$ Int. Workshop on Water Waves and Floating Bodies, Jeju, Korea., 112-115.

Korobkin, A. A., and Malenica, S. (2007). "Steep wave impact onto elastic wall." Proc., 22nd Int. Workshop on Water Waves and Floating Bodies, International Workshop on Water Waves and Floating Bodies, Oslo, Norway.

Liu, J., Guo, A., and Li, H. (2016). "Analytical solution for the linear wave diffraction by a uniform vertical cylinder with an arbitrary smooth cross-section." Ocean Eng., 126, 163-175.

Lykke Andersen, T., Frigaard, P., Damsgaard, M. L., and De Vos, L. (2011). "Wave run-up on slender piles in design conditions-Model tests and design rules for offshore wind." Coastal Eng., 58(4), 281-289.

MacCamy, R. C., and Fuchs, R. A. (1954). "Wave forces on piles: A diffraction theory." Rep. No. 69, U.S. Army Corps of Engineers, Beach Erosion Board, Washington, DC.

Mansour, A. M., Williams, A. N., and Wang, K. H. (2002). "The diffraction of linear waves by a uniform vertical cylinder with cosine-type radial perturbations." Ocean Eng., 29(3), 239-259.

Mei, C. C., Stiassnie, M., and Yue, D. K.-P. (2005). Theory and applications of ocean surface waves. Part 1: Linear aspects, vol. 23 of advanced series on ocean engineering, World Scientific Publishing Co. Pte. Ltd., Hackensack, NJ.

Mogridge, G. R., and Jamieson, W. W. (1976). "Wave forces on square caissons." Proc., 15th Int. Conf. on Coastal Engineering, 133, ASCE, Reston, VA, 2271-2289. 
Thorne, R. C. (1953). "Multipole expansions in the theory of surface waves." Math. Proc. Cambridge Philos. Soc., 49(4), 707-716.

Ursell, F. (1950). "Surface waves on deep water in the presence of a submerged circular cylinder I." Math. Proc. Cambridge Philos. Soc., 46(1), $141-152$.

Wang, Y., Ren, X., Dong, P., and Wang, G. (2011). "Three-dimensional numerical simulation of wave interaction with perforated quasi-ellipse caisson." Water Sci. Eng., 4(1) 46-60.

Williams, A. N. (1985). "Wave forces on an elliptic cylinder." J. Waterway, Port, Coastal, Ocean Eng., 10.1061/(ASCE)0733-950X(1985)111: 2(433), 433-449.
Wrobel, L. C., Sphaier, S. H., and Esperança, P. T. T. (1985). "Propagation of surface waves." Topics in boundary element research, Vol. 2, Springer, Berlin, 156-190.

Wu, X.-J., and Price, W. G. (1991). "Evaluation of wave drift forces on vertical cylinders of arbitrary geometry, with application to tension leg platforms (TLPs)." Ocean Eng., 18(1), 1-15.

Yeung, R. W. (1981). "Added mass and damping of a vertical cylinder in finite-depth waters." Appl. Ocean Res., 3(3), 119-133.

Zhu, S., and Moule, G. (1994). "Numerical calculation of forces induced by short-crested waves on a vertical cylinder of arbitrary cross-section." Ocean Eng., 21(7), 645-662. 